\title{
UNCERTAINTY MANAGEMENT IN SEISMIC VULNERABILITY ASSESSMENT USING GRANULAR COMPUTING BASED ON COVERING OF UNIVERSE
}

\author{
Khamespanah, F. ${ }^{\mathrm{a}}$, M. R. Delavar ${ }^{\mathrm{b}}$ and M. Zare ${ }^{\mathrm{c}, \mathrm{b}}$ \\ ${ }^{a}$ MSc. Student, Dept. of Surveying and Geomatics Eng., College of Eng., University of Tehran, Iran; \\ fa_khamespanah@ut.ac.ir \\ ${ }^{\mathrm{b}}$ Center of Excellence in Geomatics Eng. in Disaster Management, Dept. of Surveying and Geomatic Eng., \\ College of Eng.,University of Tehran; mdelavar@ut.ac \\ ${ }^{\mathrm{c} S e i s m o l o g y}$ Research Center, International Institute of Earthquake Engineering and Seismology (IIEES), \\ Tehran, Iran; mzare@iiees.ac.ir
}

KEY WORDS: Seismic Vulnerability Assessment, Granular Computing Model, Multi Criteria Decision Making

\begin{abstract}
:
Earthquake is an abrupt displacement of the earth's crust caused by the discharge of strain collected along faults or by volcanic eruptions. Earthquake as a recurring natural cataclysm has always been a matter of concern in Tehran, capital of Iran, as a laying city on a number of known and unknown faults. Earthquakes can cause severe physical, psychological and financial damages. Consequently, some procedures should be developed to assist modelling the potential casualties and its spatial uncertainty. One of these procedures is production of seismic vulnerability maps to take preventive measures to mitigate corporeal and financial losses of future earthquakes. Since vulnerability assessment is a multi-criteria decision making problem depending on some parameters and expert's judgments, it undoubtedly is characterized by intrinsic uncertainties.

In this study, it is attempted to use Granular computing $(\mathrm{GrC})$ model based on covering of universe to handle the spatial uncertainty. Granular computing model concentrates on a general theory and methodology for problem solving as well as information processing by assuming multiple levels of granularity. Basic elements in granular computing are subsets, classes, and clusters of a universe called elements. In this research $\mathrm{GrC}$ is used for extracting classification rules based on seismic vulnerability with minimum entropy to handle uncertainty related to earthquake data. Tehran was selected as the study area. In our previous research, Granular computing model based on a partition model of universe was employed. The model has some kinds of limitations in defining similarity between elements of the universe and defining granules. In the model similarity between elements is defined based on an equivalence relation. According to this relation, two objects are similar based on some attributes, provided for each attribute the values of these objects are equal.

In this research a general relation for defining similarity between elements of universe is proposed. The general relation is used for defining similarity and instead of partitioning the universe, granulation is done based on covering of universe. As a result of the study, a physical seismic vulnerability map of Tehran has been produced based on granular computing model. The accuracy of the seismic vulnerability map is evaluated using granular computing model based on covering of universe. The comparison between this model and granular computing model based on partition model of universe is undertaken which verified the superiority of the GrC based on covering of the universe in terms of the match between the achieved results with those confirmed by the related experts' judgments.
\end{abstract}

\section{INTRODUCTION}

One of the most well-known risks affecting urban areas is earthquake. Earthquake can be described as a 'Vibration of the earth occurred by transmission of seismic wave from the source of elastic strain energy (Talebian and Jacson, 2004).Earthquakes are not avoidable ,however, we can considerably lessen casualties by trying to model earthquake vulnerability in order to eliminate some contributing parameters in high risk regions. Tehran, capital of Iran, is among the most seismic vulnerable areas of world that is laid on known and unknown faults. According to strategic condition of this mega city, preparing fundamental information as well as vulnerability and rescue map for preparedness and mitigation of the disaster, before accruing the event is required.

One of the best methods to achieve this goal is producing a seismic vulnerability map which generally depends on various criteria. In this paper, earthquake intensity in terms of MMI unit (Modified Mercalli Intensity scale), slope, percentages of weak buildings less than 4 floors, percentage of more than 4 floor buildings, percentage of buildings built before 1966, percentage of buildings built between 1966 and 1988 is considered as effective criteria for seismic vulnerability assessment (Aghataher et al., 2005; Alinia et al., 2010; Amiri et al., 2007; Khamespanah et al, 2013; Silavi et al., 2006a, b, 2008).

In this research granular computing $(\mathrm{GrC})$ is used for extracting classification rules based on seismic vulnerability with minimum entropy. GrC is a multi-disciplinary study of problem solving and information processing that provides a general, systematic and natural way to analysis, understand, represent, and solve real world problems (Lin, 1997a; Yao, 2004, 2005; Zadeh, 1997; Chen and Yao, 2006). In this research GrC model based on neighborhood systems concept is used for defining similarity between elements based on a general relation. In this model, for each elements of the universe, a non-empty family of subset is associated that is called neighborhood system of the element, and each subset is called a neighborhood of the element (Lin, 1997a,b; Yao and Chen, 1997). The objective of this paper is to produce seismic vulnerability map of north of Tehran based on GrC model. This area is divided into 875 statistic units. The paper follows by describing the 
neighborhood systems concept in Section 2. Neighborhood systems and granular computing model are elaborated in Section 3. Research methodology is presented in Section 4. Finally, the conclusion of the paper is presented in Section 5.

\section{NEIGHBORHOOD SYSTEMS CONCEPT}

For the first time, Sierpenski and Krieger presented the theory of neighbourhood systems for the study of F'echet (V) spaces (Sierpenski and Krieger, 1956). The main concept of neighbourhood systems is explained below.

For an element $\mathrm{x}$ which is a member of a finite universe $\mathrm{U}$, we may define a subset of $\mathrm{U}$ like $\mathrm{n}(\mathrm{x}) \subseteq \mathrm{U}$ is called the neighbourhood of $\mathrm{x}$. Elements in a neighbourhood of $\mathrm{x}$ may have indistinguishability, similarity, or functionality relation with $\mathrm{x}$ (Yao, 1998).

A non-empty family of neighbourhoods of $\mathrm{x}$ which is used to explain general kinds of relationships among components of a universe of $\mathrm{x}$ is called a neighbourhood system NS(x)(Lin, 1998; Yao, 1999). A neighbourhood system of $x(\mathrm{NS}(\mathrm{x}))$ divides the universe into classes. Different neighbourhoods of $x$ contain elements having various degrees of similarity to $\mathrm{x}$. A neighbourhood system projects every element to a family of subsets of the universe, and it can be considered as an operator from $\mathrm{U}$ to $2^{2 \mathrm{U}}$.

We can show the neighbourhood system of $x$ by $\mathrm{NS}(\mathrm{x})=$ $\left\{(x)_{R}\right\}$. If $R$ is a reflexive relation, obtains a reflexive neighbourhood system which is the covering $U / R$, and if $R$ is an equivalence relation, the neighbourhood system of $\mathrm{x}$ is the equivalence class, which is the partition U/R (Lin, 1997a).

Another kind of neighbourhood system can be defined by using a distance named $D$. In this approach, $U$ is defined as a universe and $\mathrm{D}$ as a distance function. It is supposed that

$: \mathrm{U} \times \mathrm{U} \rightarrow \mathrm{R}+$, in which $\mathrm{R}+$ is the set of non-negative real numbers. For every number like $d$ which is a member of $R+$, the neighbourhood of $\mathrm{x}$ (as a member of $\mathrm{U}$ ) is defined as $\operatorname{nd}(x)=\{y \mid D(x, y) \leq d\}$ and $N S(x)=\{n d(x) \mid d \in R+\}$ is given for a neighbourhood system of $x$ (Lin, 1997b; 1998)

\section{NEIGHBORHOOD SYSTEMS AND GRANULAR COMPUTING MODEL}

In this section, based on neighbourhood systems concept, granular computing model is discussed:

\subsection{Concepts and Granules in Neighbourhood Systems}

Every concept is formally understood as a piece of thoughts that is made up of two parts of extension and intension. (Orlowska, 1987; Wille, 1992). Extension includes items which have the same characteristics that describe the concept. In the other word, that part of concept named extension is the set of entities which are its examples. The intension contains the whole attributes that are acceptable for the entire entities. Accordingly, a concept is described both by its extension and intension parts and this design helps to investigate formal concepts in a set-theoretic structure. Every subset of universe U like A can be considered as the extension of specific concepts, and members of A are described by specific properties which are the intension of the concepts. Regarding the concept A, for each elements of universe like $\mathrm{x}, \mathrm{NS}(\mathrm{x})$ is defined as Eq. 1

$\mathrm{NS}_{\mathrm{A}}(\mathrm{x})=\left\{\begin{array}{lc}\{A\} & x \in A \\ \{\varnothing\} & x \notin A\end{array}\right.$
Eq. 1 means that, the neighbourhood system of all elements not exist in A, is empty set and the set A is the neighbourhood system of every element exists in A. It is simply verified that set-theoretic operations can be considered as neighbourhood system operations by using such a representation. In addition, A should be treated as a granule since those elements in A are gathered together by their common characteristics, which means they are all examples of a definite concept.

Based on styles of definitions, a concept of different models for granular computing is formed. In this research, neighbourhood systems are employed for defining the concept, and the idea behind granular computing is discussed as follow:

In a simple $\mathrm{GrC}$ model, a finite set of attributes describe a finite set of objects named the universe presented in Eq. 2 (Pawlak, 1997; Yao, 2006; Yao and Zhong, 2002)

$\mathrm{S}=(\mathrm{U}, \mathrm{At}, \mathrm{L},\{\mathrm{Va} \mid \mathrm{a} \in \mathrm{At}\},\{\mathrm{Ia} \mid \mathrm{a} \in \mathrm{At}\})$

where,

$\mathrm{U}$ is a finite non-empty set of objects,

At is a finite non-empty set of attributes,

$\mathrm{L}$ is a language defined by using attributes in At,

$\mathrm{Va}$ is a non-empty set of values of $\mathrm{a} \in \mathrm{At}$,

Ia : $\mathrm{U} \rightarrow \mathrm{Va}$ is an information function that maps an object of $U$ to exactly one possible value of attribute a in $\mathrm{Va}$.

According to this model of $\mathrm{GrC}$, for describing granules and defining their relationships, some rudimentary formulas are applied in this study.

\subsubsection{Generality}

The generality of concept $\Phi$ is defined as the relative size of constructive granule of the concept (Talebian and Jacson, 2004) which is shown in Eq. 3 (Yao, 2001, 2008).

$\mathrm{G}(\Phi) \stackrel{\lfloor\mathrm{m}(\Phi)]}{\|\mathrm{U}\|}$

where $|\mathrm{m}(\Phi)|$ is the size of constructive granule of concept $\Phi$ and $|\mathrm{U}|$ is the size of constructive granule of universe.

\subsubsection{Absolute support}

For two given concepts $\Phi$ and $\Psi$, The absolute support (AS) or confidence of $\Psi$, provided by $\Phi$ is defined by Eq. 4 (Yao and Zhong, 2002; Yao, 2008)

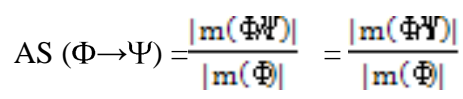

where $|\mathrm{m}(\Phi n \Psi)|$ is the size of constructive granule of concepts $\Phi$ and $\Psi_{x}$ and $|\mathrm{m}(\Phi)|$ is the size of constructive granule of concept $\Phi$.

The quantity, $0 \leq \mathrm{AS}(\Psi \mid \Phi) \leq 1$, shows the degree to which $\Phi$ implies $\Psi$.

\subsubsection{Coverage}

The coverage $\Psi$ provided by $\Phi$ is defined by Eq. 5 (Yao and Zhong, 2002; Yao, 2008)

$\mathrm{CV}(\Phi \rightarrow \Psi)=\frac{\| \mathrm{m}(\Phi)]}{\|\mathrm{m}(\Psi)\|}=\frac{\|\mathrm{m}(\Phi \Psi)\|}{\|\mathrm{m}(\Psi)\|}$ 
where $\left|\mathrm{m}\left(\Phi_{n} \Psi\right)\right|$ is the size of constructive granule of concept $\Phi$ and $\Psi_{x}$ and $|\mathrm{m}(\Psi)|$ is the size of constructive granule of concept $\Psi$.

This quantity display the conditional probability of a randomly selected object satisfying both $\Phi$ and $\Psi$

\subsubsection{Change of Support}

Change of support (CS) of concept $\Psi$ provided by concept $\Phi$ is defined by Eq. 6 (Yao and Zhong, 2002; Yao, 2008)

$\mathrm{CS}(\Psi \mid \Phi)=\mathrm{AS}(\Psi \mid \Phi)-\mathrm{G}(\Psi)$

where $\mathrm{G}(\Psi)$ may be considered as a prior probability of $\Psi$ and AS $(\Psi \mid \Phi)$ as a posterior probability of $\Psi$. The difference of prior and posterior probabilities is defined as the change of support and varies from -1 to 1 .

The positive value, shows $\Phi$ causes $\Psi$ and negative value shows $\Phi$ does not cause $\Psi$

\subsubsection{Conditional Entropy}

Consider now a family of formulas $\Psi=\left\{\Psi_{1}, \Psi_{2}, \ldots, \Psi_{\mathrm{n}}\right\}$ which induces a partition $\pi(\Psi)=\left\{\mathrm{m}\left(\Psi_{1}\right), \ldots, \mathrm{m}\left(\Psi_{\mathrm{n}}\right)\right\}$ of the universe. For formulas $\Phi$, the conditional entropy $\mathrm{H}(\Psi \mid \Phi)$ that shows the uncertainty of formulas $\Phi$ based on formulas $\Psi$ is defined by Eq. 7 (Yao and Zhong, 2002; Yao, 2008)

$H(\Psi \mid \Phi)=-\sum_{\mathrm{i}=1}^{\mathrm{n}} \mathrm{p}\left(\Psi_{\mathrm{i}} \mid \Phi\right) \log \left(\mathrm{p}\left(\Psi_{\mathrm{i}} \mid \Phi\right)\right)$

where: $\mathrm{p}\left(\Psi_{\mathrm{i}} \mid \Phi\right)=\frac{\left.\mid \mathrm{m}\left(\Phi^{\mathrm{W}} \mathrm{i}\right)\right]}{\| \mathrm{m}\left(\Phi_{2} \|\right.}$

If $\Phi$ be a certain formula, $\left(\mathrm{p}\left(\Psi_{1} \mid \Phi\right)=1\right.$ and $\mathrm{p}\left(\Psi_{j} \mid \Phi\right)=0 \forall \mathrm{j} \in 1$ : $\mathrm{n}$ and $\mathrm{j} \neq \mathrm{i}$ ), entropy reaches the minimum value, 0 .

The basic concept of granular computing model, the granular tree is composed as a flowchart shown in Figure 1

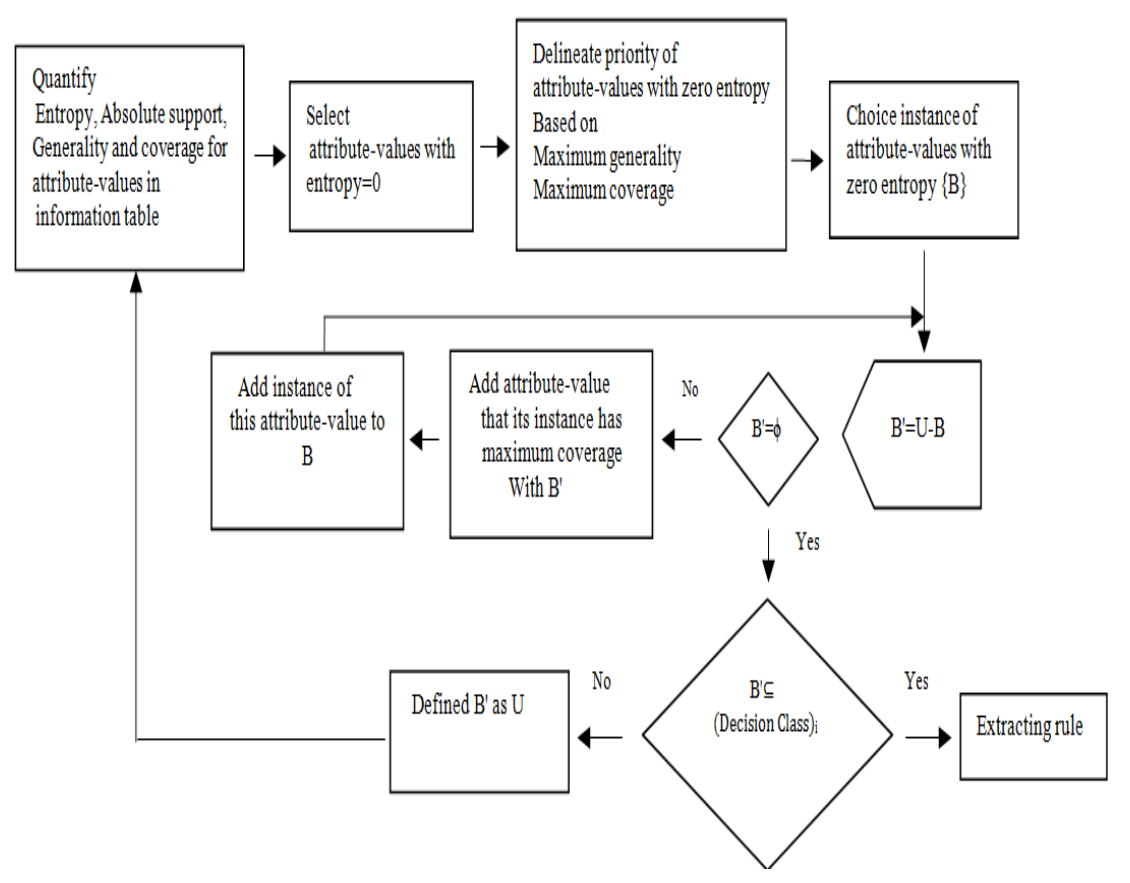

Figure 1. Granule tree algorithm for mining classification rules (Khamespanah et al., 2013)

\section{METHODOLOGY}

Data related to north of Tehran provided by census bureau of Iran and divided into 875 statistic units, were acquired. Seismic vulnerability is assessed based on activation of north Tehran fault and activation of other faults is ignored. From these 875 statistic units, a set of 30 samples is used as input for forming granular tree. For determining physical seismic vulnerability for each sample urban statistical units, experts were asked to define the physical seismic degree of vulnerability for sample statistical units by using numbers between one to four, so that 1 , 2, 3 and 4 are respectively considered as low vulnerability, medium vulnerability, high vulnerability and very high vulnerability. The information of random selection of 30 records is shown in Table1. In Table 1, seismic parameters are summarized as below (Alinia and Delavar, 2011):
Slop: Slope

MMI: MMI

Les4flor: Percentage of weak buildings less than or equal to 4 floors

Bef-66: Percentage of buildings built before 1966

Bet-66-88: Percentage of buildings built between 1966 and 1988

Up5flor: Percentage of weak buildings more than or equal to 5 floors

In this research in the first step, granular computing model based on neighbourhood systems is used. Based on information presented in Table 1, the attribute values used in constructing granule tree are considered as overlapping intervals. Classes is defined by numbers $1,2,3$ and 4 that 1 represent low value, 2 shows medium value, 3 shows high value and 4 shows very high seismic vulnerability value. The intervals correspondents to attribute values are shown as below: 
Slope

\begin{tabular}{|c|c|c|}
\hline Class & Upper limit & Lower limit \\
\hline 1 & 4 & 0 \\
\hline 2 & 13 & 3.5 \\
\hline 3 & 25 & 11 \\
\hline 4 & 45 & 21 \\
\hline
\end{tabular}

\section{MMI}

\begin{tabular}{|c|c|c|}
\hline Class & Upper limit & Lower limit \\
\hline 1 & 7.75 & 7.28 \\
\hline 2 & 8.05 & 7.65 \\
\hline 3 & 8.4 & 7.9 \\
\hline 4 & 8.98 & 8.3 \\
\hline
\end{tabular}

Percentage of Weak buildings less than or equal to 4 floors

\begin{tabular}{|c|c|c|}
\hline Class & Upper limit & Lower limit \\
\hline 1 & 22 & 0 \\
\hline 2 & 48 & 20 \\
\hline 3 & 77 & 42 \\
\hline 4 & 100 & 70 \\
\hline
\end{tabular}

\section{Percentage of buildings built before 1966}

\begin{tabular}{|c|c|c|}
\hline Class & Upper limit & Lower limit \\
\hline 1 & 10 & 0 \\
\hline 2 & 30 & 7 \\
\hline 3 & 55 & 25 \\
\hline 4 & 100 & 50 \\
\hline
\end{tabular}

\section{Percentage of buildings built between 1966 and 1988}

\begin{tabular}{|c|c|c|}
\hline Class & Upper limit & Lower limit \\
\hline 1 & 37 & 0 \\
\hline 2 & 60 & 33 \\
\hline 3 & 80 & 55 \\
\hline 4 & 100 & 75 \\
\hline
\end{tabular}

Percentage of weak buildings more than or equal to 5 floors

\begin{tabular}{|c|c|c|}
\hline Class & Upper limit & Lower limit \\
\hline 1 & 11 & 0 \\
\hline 2 & 31 & 8 \\
\hline 3 & 62 & 27 \\
\hline 4 & 100 & 58 \\
\hline
\end{tabular}

In this research attribute value classes define the concepts. Neighbourhoods of each element of universe are defined based on Eq. 1. Considering the fact that attribute values are considered as overlapping, granules are defined based on the neighbourhood systems concept. The granules are made based on similarity relation and uncertainty is effectively handled in boundaries. Similarity is a more generalized relation compared to indistinguishably relation which is used in granular computing model based on equivalence relation. By considering overlapping values, the neighbourhood defined based on similarity between objects having characteristics of reflexivity, and symmetry, but do not have transitivity characteristic. As according to this relation, a neighbourhood of $\mathrm{x}$ includes $\mathrm{x}$ itself, and for two objects of $\mathrm{x}, \mathrm{y}$, if $\mathrm{y}$ is a member of neighbourhood of $x$ (ye $n(x)$ ), then $x$ is a member of neighbourhood of y (xє y(x)). However, if yє $\mathrm{n}(\mathrm{x})$, yє $\mathrm{n}(\mathrm{z})$, then $x$ is not necessarily a member of $n(z)$ or $n(x)$ (Lin, 1997a,b).

Table 1. Information table of 30 randomly selected statistical units of Tehran

\begin{tabular}{|c|c|c|c|c|c|c|c|}
\hline S-num & $\begin{array}{c}\text { Slope } \\
(\mathbf{d e g r e e})\end{array}$ & MMI & $\begin{array}{c}\text { Les4flor } \\
(\boldsymbol{\%})\end{array}$ & $\begin{array}{c}\text { Bef66 } \\
(\boldsymbol{\%})\end{array}$ & $\begin{array}{c}\text { Bet66-88 } \\
(\boldsymbol{\%})\end{array}$ & $\begin{array}{c}\text { Up5flor } \\
(\boldsymbol{\%})\end{array}$ & Class \\
\hline 1 & 33.6 & 8.5 & 35 & 7 & 19 & 0 & 4 \\
\hline 2 & 24.5 & 8.5 & 64 & 8 & 69 & 7.65 & 5 \\
\hline 3 & 1 & 8.1 & 49 & 2 & 77 & 0 & 4 \\
\hline 4 & 5.25 & 8.1 & 29 & 19 & 55 & 0.56 & 5 \\
\hline 5 & 3.75 & 8.0 & 17 & 39 & 46 & 28.92 & 5 \\
\hline 6 & 8.7 & 8.0 & 2 & 1 & 77 & 1.86 & 4 \\
\hline 7 & 8.5 & 8.4 & 25 & 30 & 32 & 9.17 & 4 \\
\hline 8 & 3.75 & 8.8 & 21 & 14 & 52 & 22.2 & 4 \\
\hline 9 & 3.75 & 8.8 & 26 & 19 & 44 & 4.4 & 2 \\
\hline 10 & 7.2 & 7.9 & 43 & 1 & 93 & 0 & 3 \\
\hline 11 & 0.5 & 8.2 & 55 & 71 & 29 & 0 & 5 \\
\hline 12 & 8.94 & 8.2 & 4 & 2 & 74 & 1.2 & 2 \\
\hline 13 & 1 & 8.1 & 47 & 10 & 79 & 0.95 & 4 \\
\hline 14 & 3.75 & 8.0 & 51 & 43 & 39 & 1.04 & 4 \\
\hline 15 & 29.56 & 8.2 & 1 & 0 & 19 & 0 & 2 \\
\hline 16 & 8.29 & 8.2 & 6 & 0 & 78 & 0 & 3 \\
\hline 17 & 20.6 & 8.2 & 1 & 0 & 41 & 0 & 3 \\
\hline 18 & 5.83 & 8.1 & 29 & 3 & 84 & 0 & 4 \\
\hline 19 & 1 & 8.1 & 71 & 24 & 68 & 0.76 & 4 \\
\hline 20 & 3.75 & 8.108 & 84 & 25 & 67 & 15.78 & 4 \\
\hline 21 & 1 & 8.1 & 2 & 0 & 60 & 1.55 & 3 \\
\hline 22 & 13.8 & 8.6 & 0 & 0 & 53 & 1.98 & 2 \\
\hline 23 & 2.25 & 8.1 & 61 & 14 & 66 & 14.63 & 4 \\
\hline 24 & 1 & 8 & 53 & 21 & 72 & 47.52 & 4 \\
\hline 25 & 7.49 & 7.4 & 4 & 0 & 71 & 0.9 & 3 \\
\hline 26 & 7.49 & 7.4 & 20 & 16 & 63 & 13.6 & 4 \\
\hline 27 & 0 & 7.8 & 77 & 12 & 71 & 0 & 5 \\
\hline 28 & 2.7 & 7.7 & 66 & 31 & 55 & 0.77 & 5 \\
\hline 29 & 0 & 8 & 60 & 42 & 36 & 1.8 & 4 \\
\hline 30 & 0 & 8.0 & 64 & 35 & 51 & 1.27 & 4 \\
\hline
\end{tabular}


Based on the GrC model using neighbourhood systems concept, granule tree of information Table1 is represented in Figure 2.

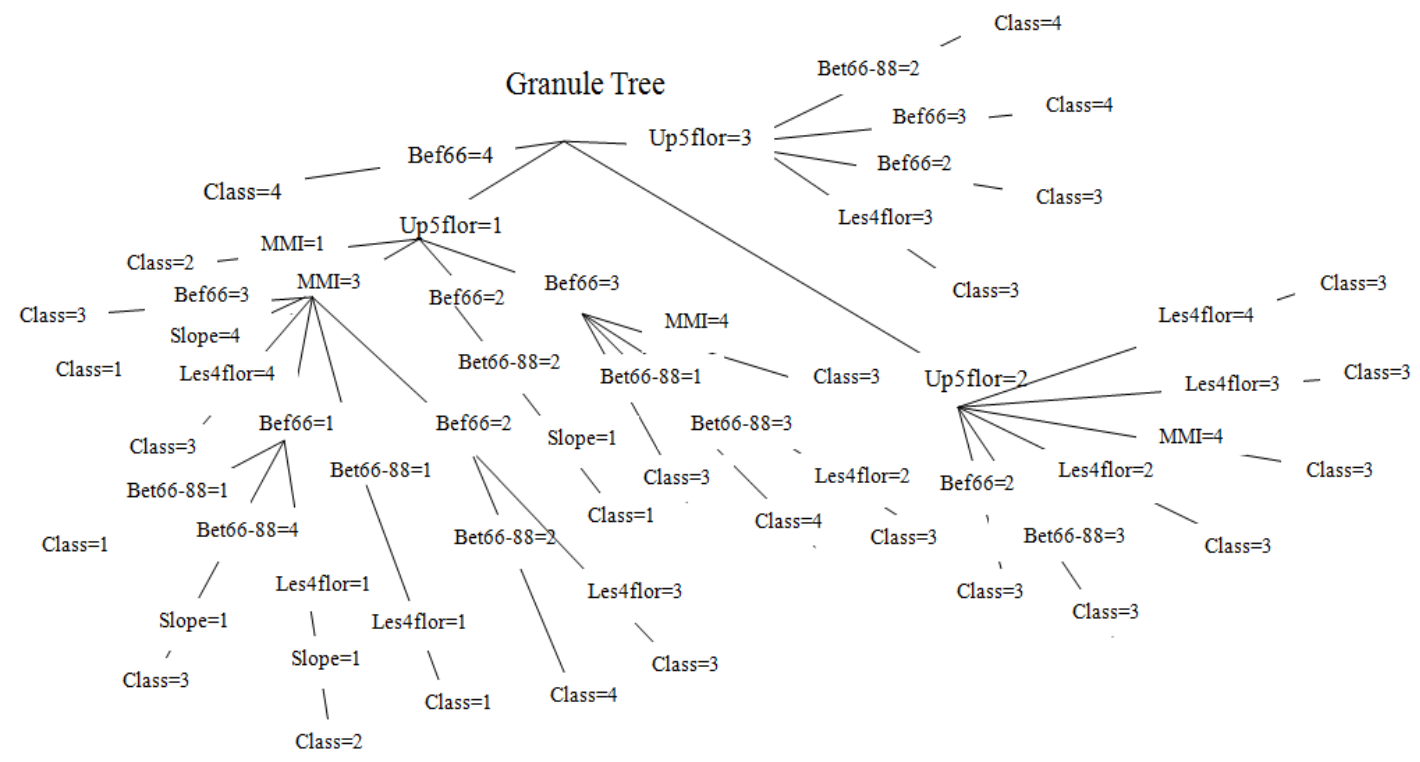

Figure 2. Granular tree based GrC model using neighbourhood systems concept

Based on the rules extracted using granule tree Figure 1, seismic vulnerability map of north of Tehran is presented in Figure 3:

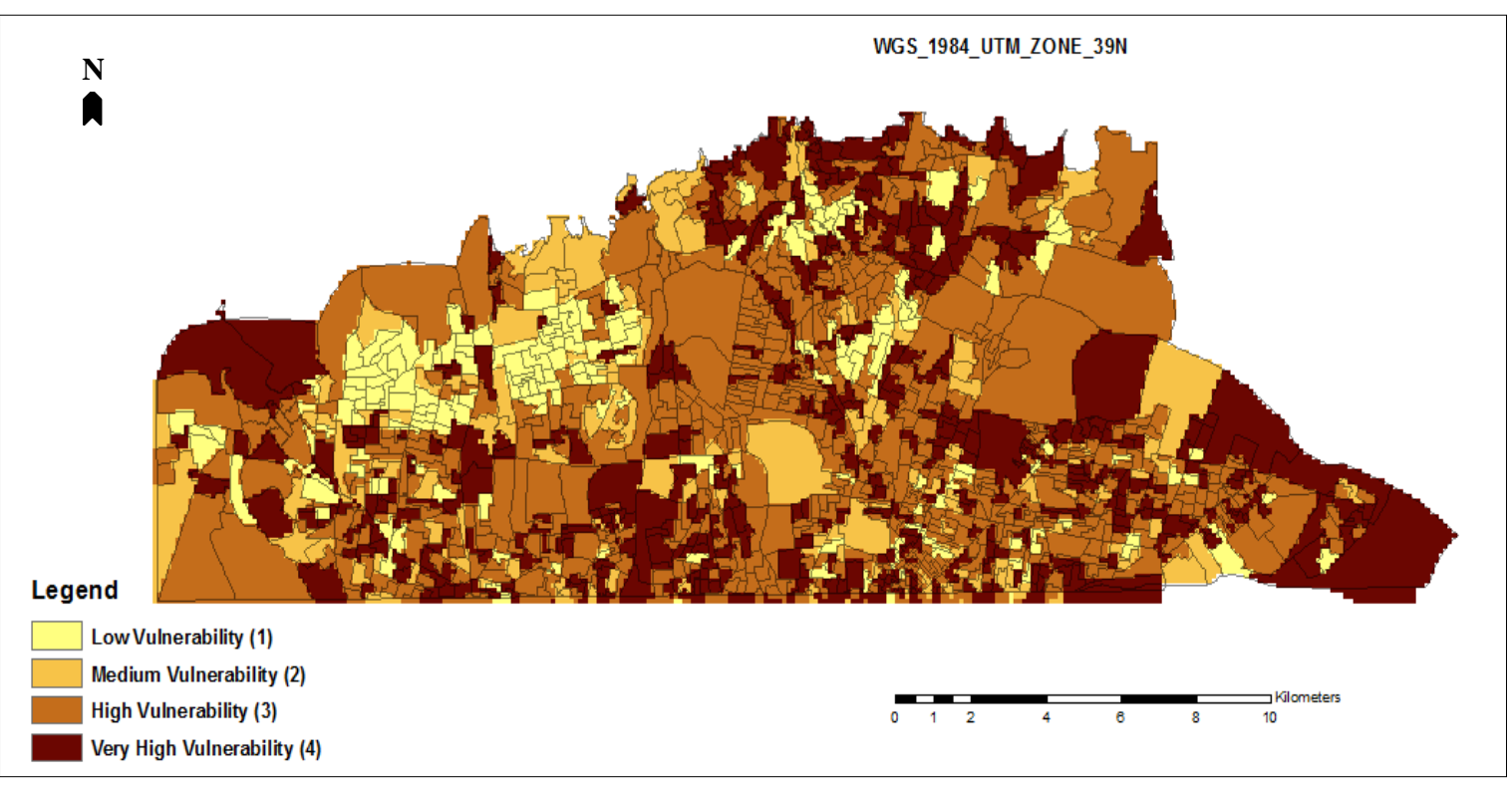

Figure 3. Seismic vulnerability map of north of Tehran based on $\mathrm{GrC}$ model using neighbourhood systems concepts

In the seismic vulnerability map provided by granular computations, $21 \%$ of the urban statistical units have low vulnerability, $18 \%$ of the urban statistical units have medium vulnerability, $45 \%$ of the urban statistical units have high level of vulnerability and $16 \%$ of the urban statistical units have very high seismic vulnerability.

\section{CONCLUSION}

Earthquake is not avoidable; however, the casualties may be considerably lessened by trying to model earthquake vulnerability in order to eliminate some contributing parameters in high risk regions. Obviously, we can extract different kinds of hidden patterns from seismic data based on nature of the gathered data, aspect of interest and purpose of study. A numbers of research, tried to determine seismic vulnerability or have a prediction of earthquake by various models such as 
partial differential equations, finite automata, or supervised learning. However, most of these approaches may not be much reliable because of low levels of accuracies. In this study, we applied GrC model based on neighbourhood systems concept for extracting rules with minimum entropy. GrC model based on equivalence relation has some kinds of limitations in defining similarity between elements of the universe and defining granules. In this model, similarity between elements is defined based on an equivalence relation. According to this

\section{REFERENCES}

Aghataher, R., M.R. Delavar, and N. Kamalian (2005), Weighing of contributing factors in vulnerability of cities against earthquakes. Proc. Map Asia Conference, Jakarta, Indonesia, Aug. 13, 2005, pp. 132- 137.

Alinia H., M.R. Delavar, and Y.Y. Yao (2010), "Support and confidence parameters to induct decision rules to classify Tehran's seismic vulnerability", Proc. The 6th International Symposium on Geo-Information for Disaster Management (Gi4DM), Torino, Italy, Sep. 15, 2010, pp. 123-130.

Alinia, H. and M.R. Delavar (2011), Tehran's seismic vulnerability classification using granular computing approach, Applied Geomatics, 3, pp. 229-240.

Amiri, A. R., M. R. Delavar, S. M. Zahrai and M.R. Malek (2007), Tehran seismic vulnerability assessment using Dempster-Shafer Theory of Evidence, Proc. Map Asia Conference 2007, Kuala Lumpur, Malaysia, Aug. 14-16,2007, 9p.

Chen, Y.H. and Y.Y. Yao (2006), Multi view intelligent data analysis based on granular computing Proc., The International Conference on Granular Computing, Georgia, USA, Sep. 23, 2006, pp. 281-286.

Khamespanah, F., M. R. Delavar, H. Samadi Alinia, and M. Zare (2013), Granular Computing and Dempster-Shafer Integration in Seismic Vulnerability Assessment, Lecture Note in Geoinformation and Cartography, Springer- Verlag Berlin Heidelberg 2013, pp.147-158.

Lin, T.Y (1997a). Granular Computing, Announcement of the BISC (Berkeley Initiative in Soft Computing) Special Interest Group on Granular Computing.

Lin, T.Y. (1997b), Neighbourhood systems - application to qualitative fuzzy and rough sets, in: Advances in Machine Intelligence and Soft-Computing, edited by P.P. Wang, Department of Electrical Engineering, Duke University, Durham, North Carolina, USA, Nov 2-5, 2007, pp.132-155.

Lin, T.Y. (1998), Granular Computing on Binary Relations I: Data Mining and Neighbourhood Systems, II: Rough Set Representations and Belief Functions, In: Rough Sets in Knowledge Discovery 1, Polkowski, L. and Skowron, A. (Eds.), Physica-Verlag, Heidelberg, pp. 107-140.

Orlowska, E. (1987), Reasoning about vague concepts, Bulletin of the Polish Academy of Sciences, Mathematics 35 p. 643-652.

Pawlak, Z. (1997), Rough set approach to knowledge-based decision support, European Journal of Operational Research, 99(1), pp. 48-57.

Sierpenski, W. and C. Krieger (1956), General Topology, University of Toronto, Toronto.

Silavi. T., M. R. Malek and M. R. Delavar, (2006a), Multicriteria map overlay in geospatial information system via intuitionistic fuzzy AHP method, Proc., The 7th International FLINZ Conference on Applied Artificial Intelligence, Geneva, Italy, Aug 29-31,2006, pp. 401-408. relation, two objects are similar based on some attributes, provided the values of these objects for each attribute be equal. In this research a general relation for defining similarity between elements of universe based on neighborhood systems concept is proposed. Based on this concept, a general relation is used for defining similarity and instead of partitioning the universe, granulation is employed using covering of universe. By using this model, uncertainty in boundaries of attributes is properly handled and more useful rules were extracted.

Silavi. T., M. R. Delavar, M. R. Malek and N. Kamalian, (2006b). An integrated strategy for GIS based fuzzy improved earthquake vulnerability assessment, Proc., The Second International Symposium on Geoinformation for Disaster Management, ISPRS, Goa, India Sep. 25-26, 2006, 5p.

Silavi. T., M. R. Malek, S. Aliabady, M. R. Delavar (2008), Dealing uncertain spatial multicriteria system via intuitionistic fuzzy method, Proc. The Joint 4th National GIS Conference and Workshop on Decision Support System, ISPRS and National Cartography Center, Tehran, Iran, Jan, 12, 2008, pp. 102-108.

Talebian, M; and J. Jackson, (2004). "A reappraisal of earthquake focal mechanisms and active shortening in the Zagros mountains of Iran". Geophysical Journal International 156 (3): pp. 506-526.

Wille, R. (1992), Concept lattices and conceptual knowledge systems, Computers Mathematics with Applications, 23, pp. 493-515.

Yao, Y.Y., (2004), A partition model of granular computing, Transactions on Rough Sets I, 3100, pp. 232-253.

Yao, Y.Y (2005). Perspectives of granular computing, Proc., IEEE Conference on Granular Computing, 1, Beijing, China, pp. 85-90.

Yao, Y.Y., and X.C. Chen (1997), Neighbourhood based information systems, Proc. The 3rd Joint Conference on Information Sciences, Volume 3: Rough Set \& Computer Science, Research Triangle Park, North Carolina, USA, March 1-5, 1997, pp. 154-157.

Yao, Y.Y. (1998), Relational interpretations of neighbourhood operators and rough set approximation operators, Information Sciences, 111, pp. 239-259.

Yao, Y.Y. (1999), Granular Computing Using Neighbourhood Systems, In: Advances in Soft Computing: Engineering Design and Manufacturing, Roy, R., Furuhashi, T., and Chawdhry, P.K. (Eds.), Springer-Verlag, London, pp. 539-553.

Yao, Y.Y. (2006), Three perspectives of granular computing, Journal of Nanchang Institute of Technology, 25, pp. 16-21.

Yao, Y.Y. and N. Zhong ( 2002), Granular Computing Using Information Tables, Studies in Fuzziness and Soft Computing, Vol. 95:pp. 102-124.

Yao, Y.Y. (2008), A Unified Framework of Granular Computing, In: Handbook of Granular Computing, Pedrycz, W., A. Skowron and V. Kreinovich (Editors), John Wiley \& Sons, Ltd, Publication, pp. 401-410.

Yao, Y. Y. (2001), On modelling data mining with granular computing, Proc., The 25th Annual International Computer Software and Applications Conference (COMPSAC 2001), IEEE, Chicago, U.S. Dec 23, 2001, pp. 638-643.

Zadeh, L.A. (1997). Towards a theory of fuzzy information granulation and its centrality in human reasoning and fuzzy logic, Fuzzy Sets and Systems, 19, pp. 111-127. 